Research Article

\title{
Wind Tunnel Tests of Wind-Induced Snow Distribution for Cubes with Holes
}

\author{
Xintong Jiang $\mathbb{D}$, Zhixiang Yin $\mathbb{D}$, and Hanbo Cui $\mathbb{D}$ \\ School of Mechanics and Engineering, Liaoning Technical University, Fuxin, Liaoning 123000, China \\ Correspondence should be addressed to Zhixiang Yin; lntuyzx@163.com
}

Received 5 July 2019; Revised 8 September 2019; Accepted 16 September 2019; Published 22 November 2019

Academic Editor: Pier Paolo Rossi

Copyright (c) 2019 Xintong Jiang et al. This is an open access article distributed under the Creative Commons Attribution License, which permits unrestricted use, distribution, and reproduction in any medium, provided the original work is properly cited.

\begin{abstract}
The nonuniform distribution of snow around structures with holes is extremely unfavorable for structural safety, and the mechanism of wind-snow interaction between adjacent structures with holes needs to be explored. Therefore, a wind tunnel simulation was performed, in which quartz particles with an average particle size of $0.14 \mathrm{~mm}$ as snow particles were used, and cubes with dimensions of $100 \mathrm{~mm} \times 100 \mathrm{~mm} \times 100 \mathrm{~mm}$ each containing a hole with the size of $20 \mathrm{~mm} \times 20 \mathrm{~mm}$ were employed as structures. Firstly, the quality of a small low-speed wind tunnel flow field was tested, and then the effects of hole orientation (hole located on the windward side, leeward side, and other vertical sides) and absence of holes on the surface of a single cube were studied. Furthermore, the effects of different hole locations (respectant position, opposite position, and dislocation) and relative spacing $(50 \mathrm{~mm}, 100 \mathrm{~mm}$, and $150 \mathrm{~mm}$ ) on the surfaces of two cubes and the snow distribution around them were investigated. It was concluded that the presence and location of hole had a great influence on snow distribution around cubes. Snow distribution was favorable when hole was located on the other vertical sides of the test specimen. The most unfavorable snow distribution was obtained when the holes on the two-holed sides of the cubes were respectant with a maximum snow depth coefficient of 1.4. A significant difference was observed in the snow depths of two sides of cubes when holes were dislocated. When two holes were respectant, surrounding snow depth was decreased, and the maximum snow depth on model surface area was increased with the increase of spacing. Wind tunnel tests on holed cubes provided a reference for the prediction of snow load distribution of typical structures with holes.
\end{abstract}

\section{Introduction}

In recent years, a large number of buildings with unique shapes and novel structures have appeared in cities. As a manner of architectural expression, setting up holes on external walls have widely been used by many designers, like those observed in Church on the Water, Shanghai Poly Grand Theater, Langxiang Church, and Yinhe SOHO. Setting up holes has the advantages of good lighting, environmental protection, and closeness to nature. The main structure of Shanghai Poly Grand Theater is $100 \mathrm{~m} \times 100 \mathrm{~m} \times 35 \mathrm{~m}$ in dimensions, with 5 curved holes on external walls. The hole on the eastern wall has the largest size, with a horizontal span of $45.21 \mathrm{~m}$, vertical height of $20.35 \mathrm{~m}$, and hole area of $670.83 \mathrm{~m}^{2}$. This kind of opening structure is mostly used in churches, theatres, business centers, large office areas, and other places with dense populations. The nonuniform distribution of snow on such buildings may cause deformations and even failure of the structures, threatening the lives and properties of people. Therefore, it is very important to determine the distribution rule of snow in structures with holes for safety reason.

Wind tunnel test is considered as one of the most reliable methods in studying wind-induced snow drifting. Test results could be used to verify the precision of numerical simulations. Unlike field measurements, parameters such as the speed, direction, and blowing time of wind can be controlled and adjusted in wind tunnel tests. So far, several researchers around the world have conducted a series of studies considering similar main parameters [1-4] such as rough height Reynolds and Froude numbers $[1,5,6]$, time scale [7], and other parameters. Peterka et al. [8] conducted 
wind tunnel tests on the snow distribution of model surfaces under different wind directions. O’Rourke et al. [9] studied the mechanisms of the distribution and mass transport rate of snow under three different types of roofing through wind tunnel tests. Liu et al. [10] carried out wind tunnel tests using different model sizes and wind speeds considering snow residue and erosion rates and determined the impact of snow distribution on roofing. Yu et al. [11] conducted wind tunnel tests based on 3D flat roof models to analyze the effect of model size, wind speed, and wind blowing time on snow redistribution.

Different researchers have also studied the media used to simulate snow particles. Kind [4] found that the results obtained in using heavy quartz sand particles were closer to the experimental findings than light foam particles. Zhou et al. [12] conducted wind tunnel tests using three different media: low-density saw wood ash, medium-density polyfoam, and high-density silica sand. They found that among the tested three media, high-density silica sand agreed well with measured results. Li [13], Wang et al. [14], and Zhou et al. [15] designed wind tunnel tests using quartz sand particles as snow particles to investigate wind-induced snow distributions. In the aforementioned studies, fine quartz sand particles were used as snow particles in wind tunnel tests on wind-induced snow drift.

Currently, most of the studies adopt single closed structures as research objects, and very limited studies are conducted on holed structures and holed adjacent structures. The majority of previous wind tunnel tests have mainly studied snow depth coefficient $C_{\mathrm{s}}$ along the axis line, while nonuniform snow distribution around structures also affects the normal use of doors, windows, and evacuation channels. In addition, there are very few regulations on snow load on holed structures and this leads to the surface of structures to be unsafe. Snow movement between adjacent structures with holes is more complicated than the single structure, and the snow distribution patterns are still unclear. Therefore, in order to clarify the influence of hole orientation and the distribution of snow around it, wind tunnel tests were carried out using regular holed cubes as models. This study provides a basis for predicting the snow distribution patterns in structures with holes.

\section{Small Low-Speed Wind Tunnel}

2.1. Wind Tunnel Design and Inspection. If a closed circuit type wind tunnel was used to study snow distribution patterns around structures, the prelaying material used as snow particles could cause great damage to wind blades. Therefore, a small low-speed wind tunnel was designed for wind and snow research. The constructed small low-speed wind tunnel was $7 \mathrm{~m}$ long and consisted of a test section, a stable section, a rectifier device (honeycomb), a shrink section, a diffusion section, and wind blades. The dimensions of the profile of the experiment section was $0.5 \mathrm{~m} \times 0.5 \mathrm{~m}$ with a maximum fan frequency of $50 \mathrm{~Hz}$ and a steady wind speed of $1.5 \sim 10 \mathrm{~m} / \mathrm{s}$ in the test section. A box was placed at the exit of the diffusion section to determine mass transport rate. Honeycomb was installed in the stable section, which converted the vortex into air flow parallel to the longitudinal axis of the wind tunnel; the vortex could be attenuated quickly to get even air flow. The instruments used in the wind tunnel test are shown in Figure 1.

Feasibility tests were carried out on the airflow stability, dynamic pressure field coefficient, and speed uniformity of the wind tunnel test section. Three sections $250 \mathrm{~mm}$ (a), $562.5 \mathrm{~mm}$ (b), and $875 \mathrm{~mm}$ (c) away from the entrance of the test section were selected, and each section was divided into 9 small square blocks with equal areas. The measurement points were located in the center of square blocks, as shown in Figure 2.

Dynamic pressure pulsation $\eta$ is used to describe flow stability and is defined as the pulsation of air dynamic pressure for $1 \mathrm{~min}$ :

$$
\eta=\frac{q_{\max }-q_{\min }}{q_{\max }+q_{\min }}
$$

where $q_{\max }$ and $q_{\min }$ are, respectively, the maximum and minimum values of instantaneous dynamic pressure $(\mathrm{Pa})$.

The dynamic pressure field coefficient $\mu_{i}$ reflects the spatial distribution of dynamic pressure in the flow field:

$$
\begin{aligned}
& \mu_{i}=\frac{q_{i}-\bar{q}}{\bar{q}}=\frac{q_{i}}{\bar{q}}-1, \\
& \bar{q}=\frac{1}{n} \sum_{i=1}^{n} q_{i},
\end{aligned}
$$

where $q_{i}$ is the instantaneous dynamic pressure of point $i$ $(\mathrm{Pa}), \bar{q}$ is the average value of the measured point dynamic pressure in the model $(\mathrm{Pa})$, and $n$ is the number of measured points. $\sigma_{\mathrm{v}}$ is the root mean square of the relative deviation between the flow velocity and mean velocity in the test section which can be used to describe the uniformity of velocity:

$$
\begin{aligned}
& \sigma_{v}=\sqrt{\frac{\sum_{i=1}^{n}(\Delta v / \bar{v})^{2}}{n-1}}, \\
& \bar{v}=\frac{1}{n} \sum_{i=1}^{n} v_{i}, \\
& \Delta v=v_{i}-\bar{v}
\end{aligned}
$$

in which, $\bar{v}$ is the average velocity of flow $(\mathrm{m} / \mathrm{s}), v_{i}$ is the velocity of point $i(\mathrm{~m} / \mathrm{s})$, and $\Delta v$ is the velocity deviation of point $i(\mathrm{~m} / \mathrm{s})$. The data measured through a ZC1000-1F digital micromanometer and a pitot tube were analyzed to obtain the aforementioned three parameters, as shown in Figures 3 and 4 and Table 1.

The flow field quality specifications of high-speed and low-speed wind tunnels required low-speed aviation wind tunnels to meet $\eta \leq 0.005$ and closed circuit type wind tunnels to meet $\eta \leq 0.015$. As shown in Figure 3, the dynamic pressure pulsations obtained for each measuring point in section $c$ at different frequencies met $\eta \leq 0.015$. Therefore, the airflow stability of section c satisfies general wind tunnel qualification standard. 


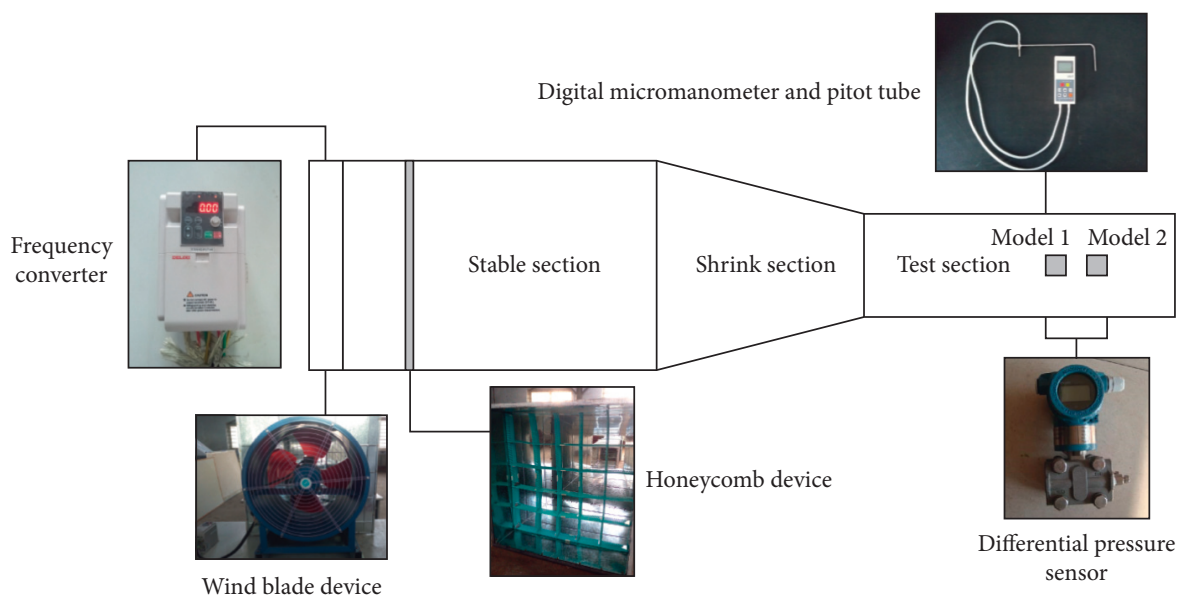

FIgURE 1: Instruments used in the wind tunnel test.

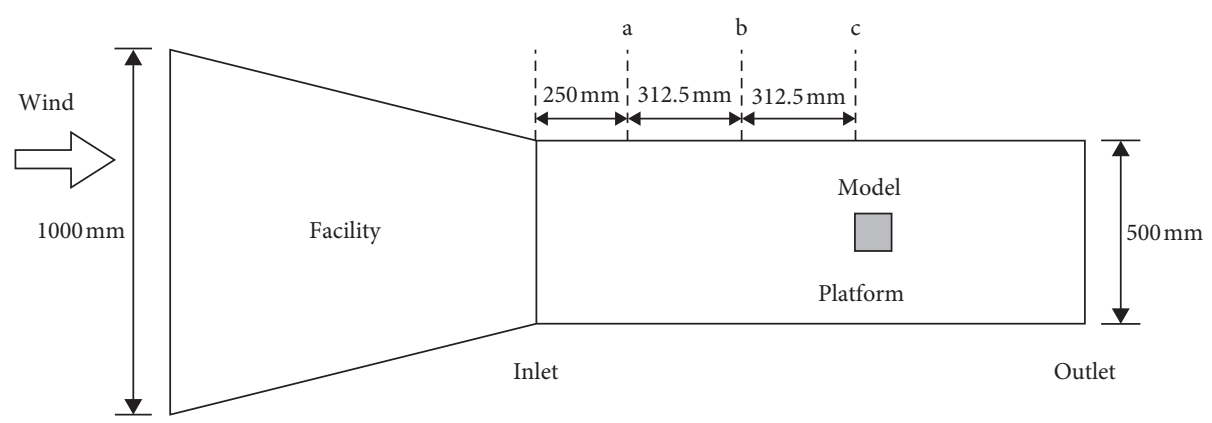

FIGURE 2: Diagram of the wind tunnel test.

The wind tunnel test standard method for building engineering requires building wind tunnels to meet $\mu_{i} \leq 2 \%$. It can be concluded from Figure 4 that the dynamic pressure field coefficient of section $c$ was qualified for the wind tunnel test section.

It was concluded from Table 1 that $\sigma_{v}$ of section c was the smallest when fan frequency was the same, and the upper limit of $\sigma_{v}$ for construction wind tunnel was $2 \%$. When the frequency was increased, the upper limit of section $c$ was increased by $35 \%, 26 \%$, and $5 \%$, approached the qualification standard. It was assumed that section $c$ met the flow field regulations of the wind tunnel test section.

Turbulent intensity $\varepsilon$ is a key parameter for the wind tunnel and is defined as the ratio of the average square root of pulsation velocity to the average velocity of the main flow along $x, y$, and $z$ directions.

$$
\varepsilon=\frac{\sqrt{(1 / 3)\left(v_{x}^{\prime 2}+v_{y}^{\prime 2}+v_{z}^{\prime 2}\right)}}{\bar{v}},
$$

where $v_{x}^{\prime}, v_{y}^{\prime}$, and $v_{z}^{\prime}$ are the pulsation values of velocity along $x$, $y$, and $z$ directions. The turbulence intensity of the wind tunnel was no larger than $1.96 \%$. The wind tunnel test standard method for building engineering requires a turbulence intensity of $\varepsilon \leq 2 \%$; therefore, our results met this requirement.

The frequency converter CDI-EM60G2R2T4B was used to adjust wind speed, and a ZC1000-1F digital micromanometer and a pitot tube were used to measure wind speed. Measurements were repeated three times at the same frequency and their average value was obtained as the results, as shown in Figure 5.

As shown in Figure 5, the wind speed increased with the increase of fan frequency, which basically showed a linear change and was fitted as shown in (5). The comparison between fitting and the measuring values are shown in Figure 6.

$$
v=0.199 f+0.837, \quad 5 \mathrm{~Hz} \leq f \leq 50 \mathrm{~Hz},
$$

where $v$ is wind speed $(\mathrm{m} / \mathrm{s})$ and $f$ is fan frequency $(\mathrm{Hz})$.

It can be seen in Figure 6 that the fitting value had the same trend with the measured values, with small differences in values and $R^{2}=0.979$. In equation (5), a lower limit of $5 \mathrm{~Hz}$ for the fan frequency was set because the uniformity of wind flow was reduced. Meanwhile, an upper limit of $50 \mathrm{~Hz}$ is set for safety reason of the equipment.

2.2. Similarity Criteria. In this test, the model is geometrically similar, and the angle of repose similarities is replaced with geometric similarities for media simulating snow particles. Smedley et al. [16] found that test results were only slightly affected by the size of snow particles. Motion similarity requires that flow velocity ratios at any point in the model and the prototype are equal; the similarity of the motion of the atmospheric boundary layer was 


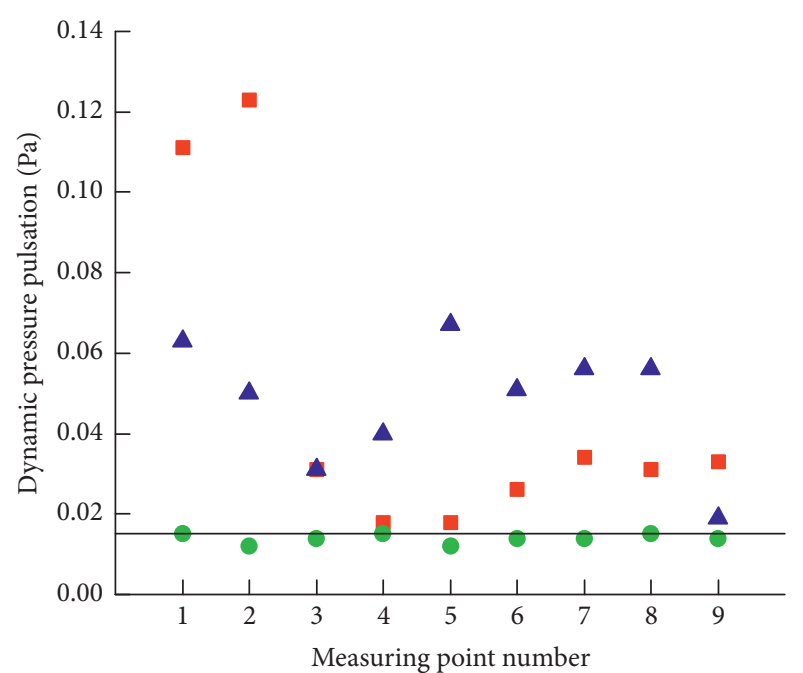

Section a

Section b

Section $\mathrm{c}$

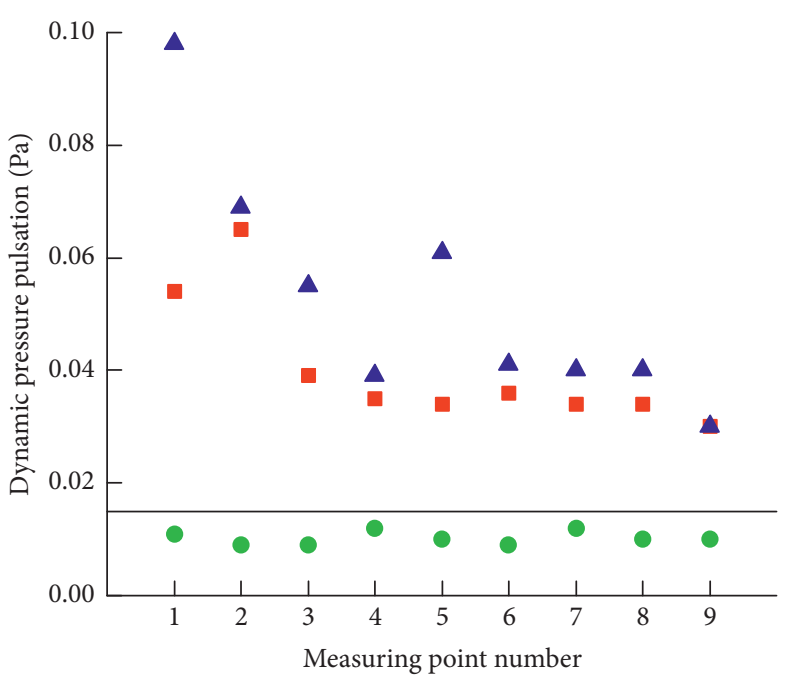

- Section a

- Section b

- Section c

(a)

(b)

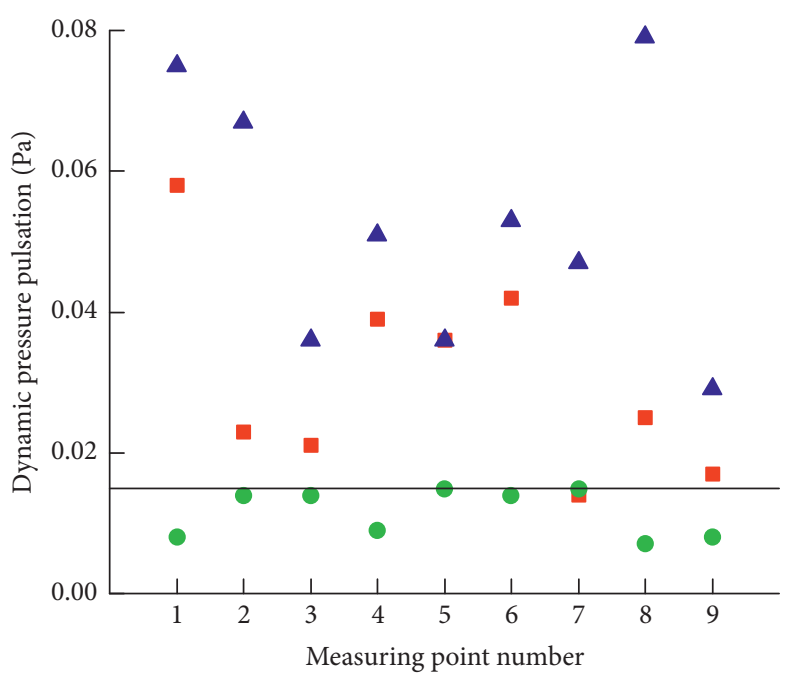

$\Delta$ Section a

- Section b

- Section c

(c)

Figure 3: Dynamic pressure pulsation under different frequencies: (a) $30 \mathrm{~Hz}$, (b) $40 \mathrm{~Hz}$, and (c) $50 \mathrm{~Hz}$.

also considered. Power similarity mainly requires similar parameters of Froude number $L g / U^{2}$ and speed ratios $u_{*} / w_{\mathrm{f}}$ and $u_{* \mathrm{t}} / u_{*}$, where $L$ is the geometric scale of the model, $g$ is the gravity, $U$ is the reference value of speed, $u_{*}$ is the friction velocity, $w_{\mathrm{f}}$ is the particle settling velocity, and $u_{* \mathrm{t}}$ is the threshold friction velocity. In the meanwhile, the Reynolds number $u_{*}^{3} / 2 g v$ also need to be considered, where $v$ is the kinematic viscosity of the fluid. Classical Reynolds and Froude numbers are contradictory. If particle density was high, Reynolds number could be satisfied, but not Froude number. Kind [4] showed the test results satisfying Reynolds number were closer to the experimentally measured values.

\section{Wind Tunnel Test}

3.1. Model and Parameter Selection. The experimental model was similar to that developed by Oikawa et al. [17] in Hokkaido University of Technology, Japan. The model used a cube with side length $1 \mathrm{~m}$ and experimental geometric scale ratio of $1 / 10$. The test cases include a single cube, a single-holed cube, and two-holed cubes. The cubes were $100 \mathrm{~mm}$ long, and the square hole was $20 \mathrm{~mm}$ high. The distance between two models $L$ was variable, as shown in Figures 7 and 8. In the experiment, hole direction in a single-holed cube as well as relative location and spacing between the two models were investigated. Quartz sand 


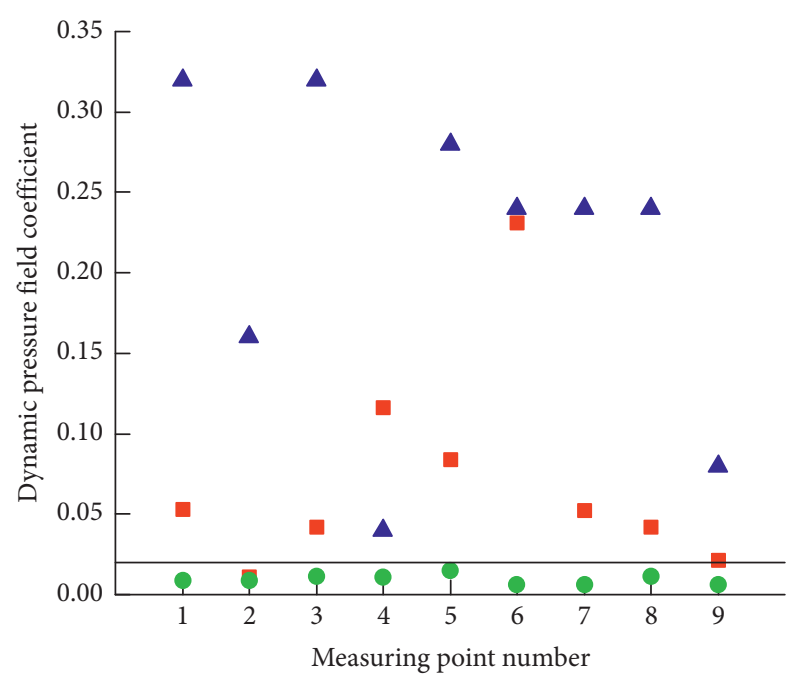

- Section a

Section b

- Section c

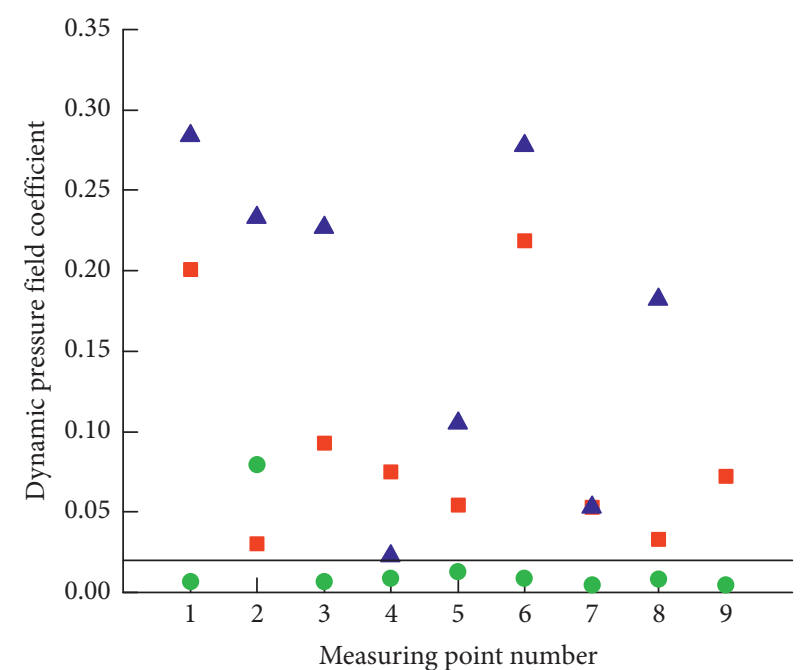

Section a

Section b

- Section c

(a)

(b)

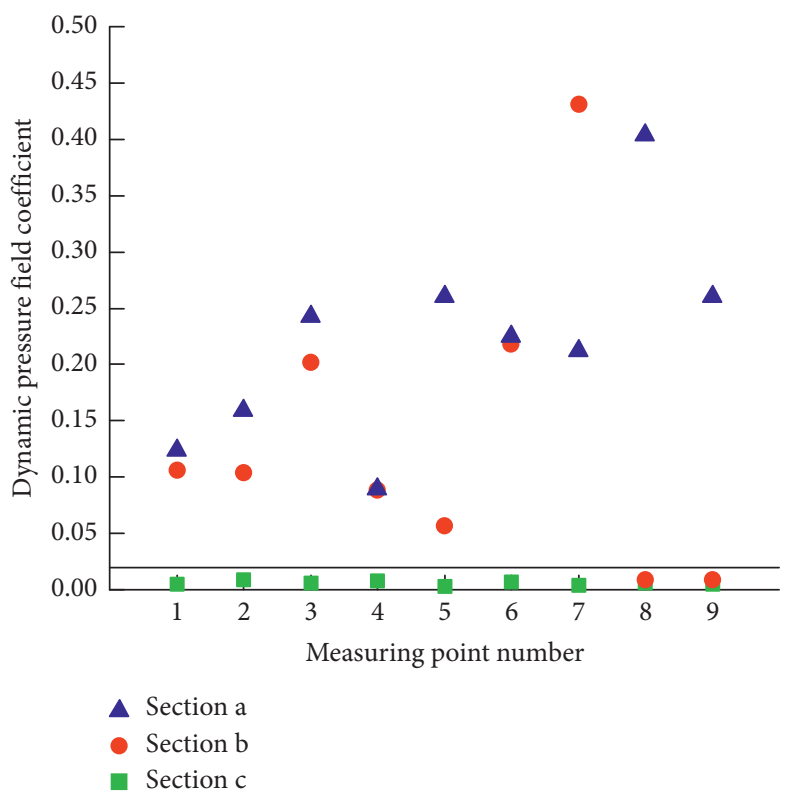

(c)

Figure 4: Dynamic pressure field coefficient under different frequencies: (a) $30 \mathrm{~Hz}$, (b) $40 \mathrm{~Hz}$, and (c) $50 \mathrm{~Hz}$.

TABLE 1: Deviation mean squares of wind speed and average speed.

\begin{tabular}{lccc}
\hline Fan frequency $(\mathrm{Hz})$ & Section a & Section $\mathrm{b}$ & Section $\mathrm{c}$ \\
\hline 30 & 0.127 & 0.05 & 0.031 \\
40 & 0.111 & 0.059 & 0.027 \\
50 & 0.13 & 0.106 & 0.021 \\
\hline
\end{tabular}

particles were used as snow particles, which have been widely used in other works as well $[13-15,18]$. The values of quartz sand particles and other parameters used in the test are summarized in Table 2. Prototype values were selected from the works of Smedley et al. [16], Beyers and Harms [19], and Kind and Murray [20].
As shown in Table 2, since $w_{\mathrm{f}}$ was larger than the prototype value, $w_{\mathrm{f}} / u_{*}$ and $w_{\mathrm{f}} / u_{* \mathrm{t}}$ were both two and three times larger than those of the prototype. The model value of Froude number was two orders of magnitude higher than that of the prototype, which is a large difference. Therefore, Froude number simulation was considered to be inaccurate, but other parameters satisfied the requirements.

3.2. Test Description. Predistribution particle method was used to simulate snow drifting. The prelaying thickness of quartz sand was assumed to be similar with the geometric scale ratio of the snow thickness measured in Oikawa 


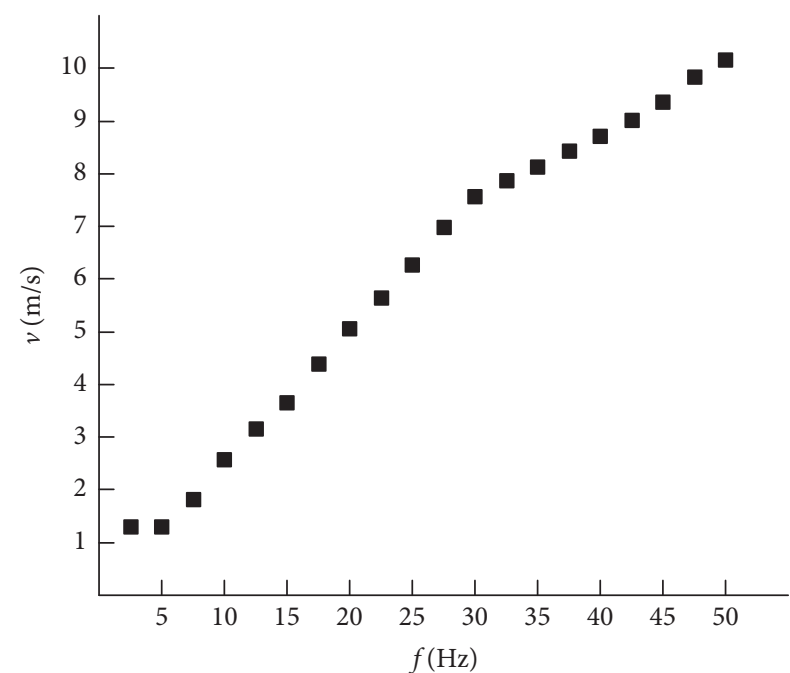

FIgURE 5: Wind speed versus frequency.

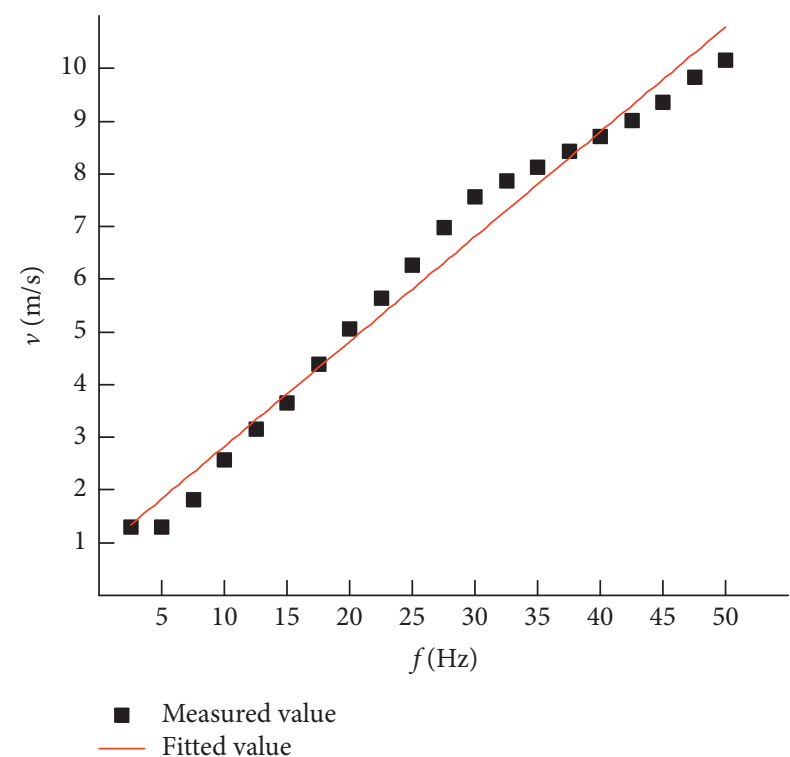

FIGURE 6: Fitting formula and measuring wind speed.

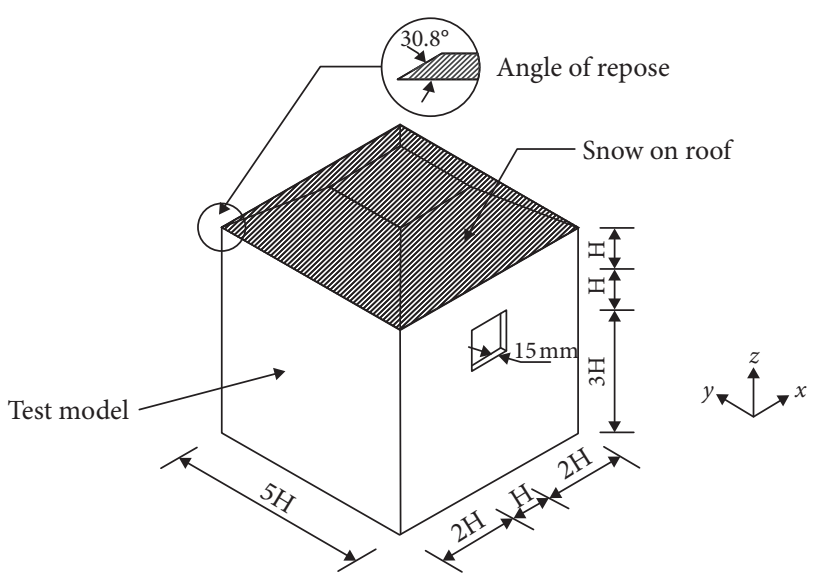

FIgURE 7: Single-holed test model.

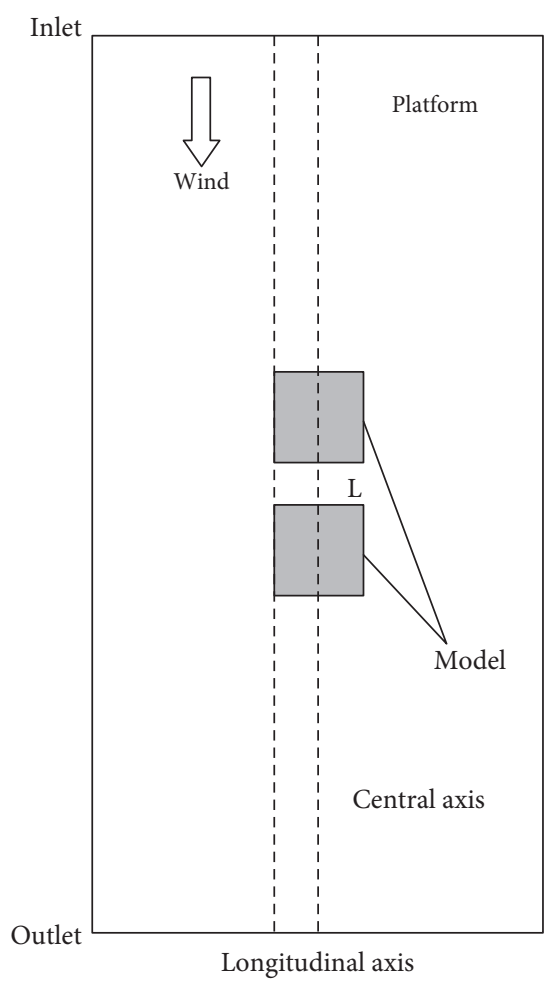

Figure 8: Two-holed test models.

TABle 2: Physical properties of particles and other main parameters.

\begin{tabular}{lcc}
\hline Parameters & Model values & Prototype values \\
\hline Mean diameter $(\mathrm{mm})$ & 0.14 & $0.1 \sim 4$ \\
Density $\left(\mathrm{g} / \mathrm{cm}^{3}\right)$ & 2.56 & $0.4 \sim 2.7$ \\
Angle of repose $\left(^{\circ}\right)$ & 30.8 & $30 \sim 50$ \\
$u_{* \mathrm{t}}(\mathrm{m} / \mathrm{s})$ & 0.14 & $0.118 \sim 0.28$ \\
$w_{\mathrm{f}}(\mathrm{m} / \mathrm{s})$ & 2 & $0.31 \sim 0.75$ \\
$u_{* \mathrm{t}}^{3} / 2 \mathrm{gv}$ & 9.66 & $7 \sim 70$ \\
$u_{*} / u_{* \mathrm{t}}$ & $1.31 \sim 1.69$ & $0.66 \sim 7.93$ \\
$w_{\mathrm{f}} / u_{*}$ & $8.44 \sim 10.91$ & $0.3 \sim 3.9$ \\
$u_{*}^{2} / \mathrm{Lg}$ & $0.013 \sim 0.02$ & $0.00043 \sim 0.00072$ \\
$w_{\mathrm{f}} / u_{* \mathrm{t}}$ & 14.28 & $2.16 \sim 5$ \\
\hline
\end{tabular}

(200 $\mathrm{mm})$, and the flow direction of the test section was covered with quartz sand particles to simulate flow boundaries. The windward side of the cube was placed at section c, and a differential pressure sensor 3051TG was set at this position. Another identical device was placed on the leeward side of model 2, as shown in Figure 1. In this way, the quality of the flow field in the test area can be detected at any time to ensure the stability of airflow. The threshold wind speed was assumed to be $3.8 \mathrm{~m} / \mathrm{s}$ when quartz sand particles were blown up and had obvious movements. Test time was determined to be 9 min according to time scale similarity. Wind speed was determined by a similarity ratio, and the measured average wind speed was $4.5 \mathrm{~m} / \mathrm{s}$. Particle depth was measured using a slide-type laser range finder. Final particle depth was considered to be dimensionless $(20 \mathrm{~mm})$ to obtain snow depth coefficient $C_{\mathrm{s}}$. To reduce random errors, each group of experiments was conducted in 
three replicates and the average value was reported. Figure 9 shows the wind-induced snow distribution test with $100 \mathrm{~mm}$ spacing between two-holed models.

\section{Test Results}

4.1. Single Cube. Horizontal axis was dimensionless with model height as the characteristic scale. Four conditions including a nonholed single cube, windward side hole, leeward side hole, and other vertical side hole were tested. The distribution curve of snow depth coefficient $C_{s}$ of the model surface and surrounding under the above-mentioned four conditions was obtained and compared with Oikawa test results. When snow depth coefficient $C_{\mathrm{s}}$ was greater than 1, quartz sand particles were deposited, and when it was below 1 , the particles were eroded, as given in Figure 10.

As shown in Figure 10(a), on the windward area, the measured $C_{\mathrm{s}}$ first decreased and then increased, and its minimum value $(0.5)$ appeared at about $x / H=-0.83$, after which it rapidly increased. On the windward area of the nonholed test model, the minimum value of $C_{\mathrm{s}}(0.55)$ appeared at about $x / H=-1.5$. Then, it gradually increased until it reached its maximum value at $x / H=-0.5$. In the leeward area, the measured data and test data had the same variation trend. Possible reasons for the difference between the measured value and the experimental value are as follows: there are trapped vortexes on the windward side of the cube at the position close to the ground, which cause the wind to flow reversely in the horizontal direction. This phenomenon leads $C_{\mathrm{s}}$ in the windward area to first decrease and then increase. The measured and tested trapped vortex areas are different, and the increase in the range of Cs on the $\mathrm{x} / \mathrm{H}$ axis is also different. Also, the fact that the wind speed was not stable and the direction of wind flow was not always perpendicular to the windward surface may contribute to the difference; Another factor is the experimental equipment difference, which has a certain influence on the $C_{\mathrm{s}}$ values. On the leeward area of the model, $C_{\mathrm{s}} \leq 1$ which was smaller than other scenarios when the hole was located on leeward and other vertical sides of the model. It was concluded that the presence and location of the hole had significant influences on the snow distribution of the model. The values of $C_{s}$ under the two conditions have minor differences. It is believed that both conditions are advantageous for the distribution of snow along the central axis.

It was seen from Figure 10(b) that two horseshoe vortexes were formed at the corner of the windward face of the model. Snow was totally eroded when $x / H=-0.75 \sim-0.4$ at $C_{\mathrm{s}}=0$. Then, $C_{\mathrm{s}}$ was sharply increased and reached its first crest at $x / H=0.25$. At this point, $C_{\mathrm{s}, \max }$ was about 0.9 1.2. The value of $C_{s}$ on the leeward area was larger than that along the central axis and changed the form of corrugations because snow was creeped at these locations. When the hole was located on the other vertical sides, the snow around the structure was eroded. Under the three mentioned conditions of the absence of hole and the hole located on the windward and leeward sides of the model, the values of $C_{\mathrm{s}}$ were $18.18 \%$,

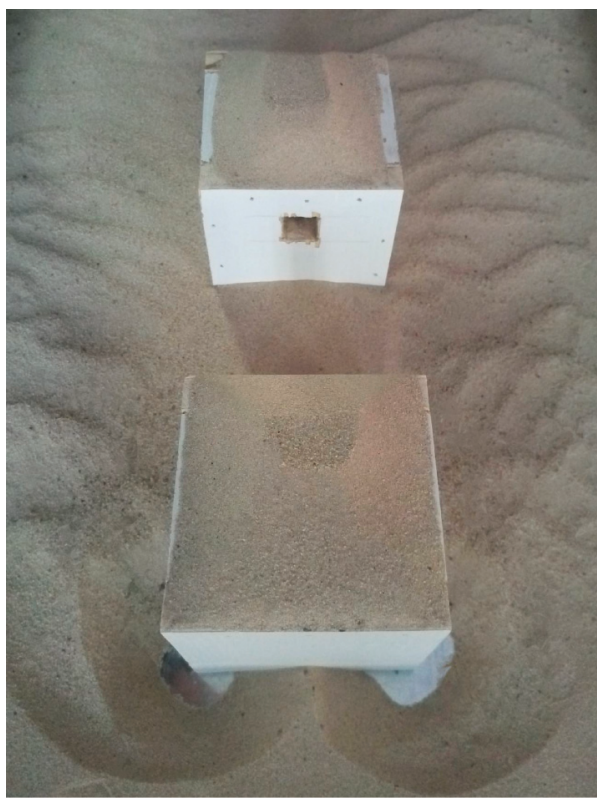

FIgURE 9: Snow distribution after test.

$18.18 \%$, and $10 \%$ larger than the corresponding values when the hole was located on the side along the longitudinal axis of the leeward edge $(x / H=-0.5)$, respectively. At $x / H=1, C_{s}$ of the hole located on the side showed the biggest difference with other three conditions (about 33.33\%, 30.43\%, and $27.27 \%$, respectively), indicating that the hole on the side had a favorable influence on snow distribution along the longitudinal axis of the model.

From Figure $10(\mathrm{c})$, the minimum value of $C_{\mathrm{s}}$ in the model roof $(0.2 \sim 0.3)$ appeared at the windward edge $(x /$ $H=-0.5)$, and roof leeward area $(x / H=0.25)$ had the largest amount of snow deposition with a value of $0.9 \sim 1$. At this point, the $C_{s}$ value of the hole located on the windward side was $10 \%$ higher than that of the cases of the absence of hole and the hole located on the other vertical sides. More attention had to be paid to the uneven distribution of snow load on the roof. Based on the aforementioned three figures, the hole located on the side gave the best distribution of snow on the surface and surrounding of a single cube.

4.2. Hole Location. The $C_{\mathrm{s}}$ values of two-holed models that are $100 \mathrm{~mm}$ away from each other, with three different hole locations of respectant position, opposite position, dislocation of the central axis, longitudinal axis, and roof were studied. Longitudinal axis was selected as shown in Figure 11.

As shown in Figures 12(a) and 12(b), the distribution patterns of snow around disturbance model (model 1) and disturbed model (model 2) were completely different. Snow movement between the two structures was extremely complicated, and the superposition of snow distribution around the two single structures is not regular. The $C_{\mathrm{s}}$ values of windward area of the models were generally smaller than those along leeward area, and a large amount of snow was 


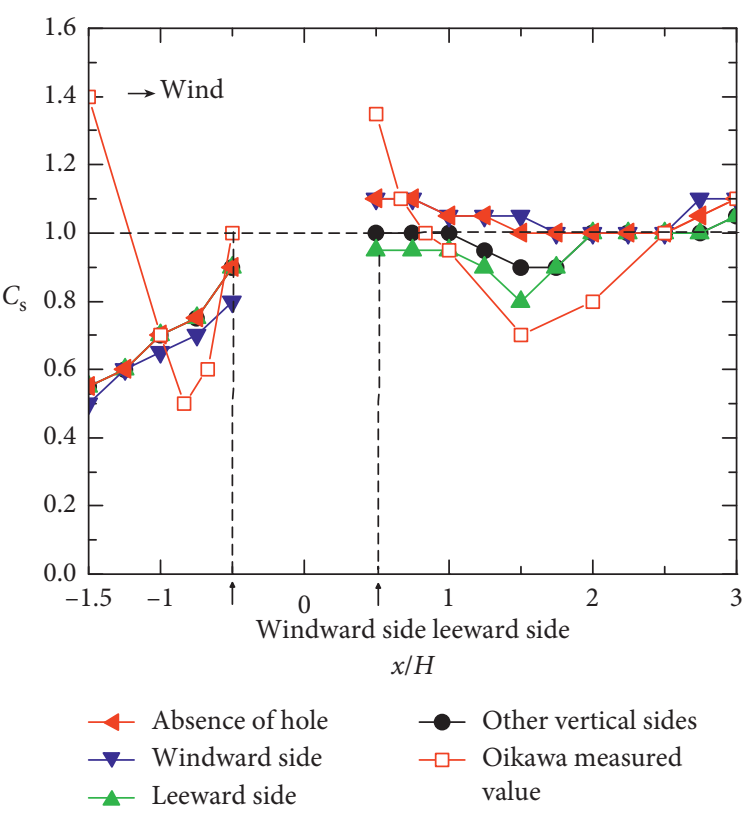

(a)

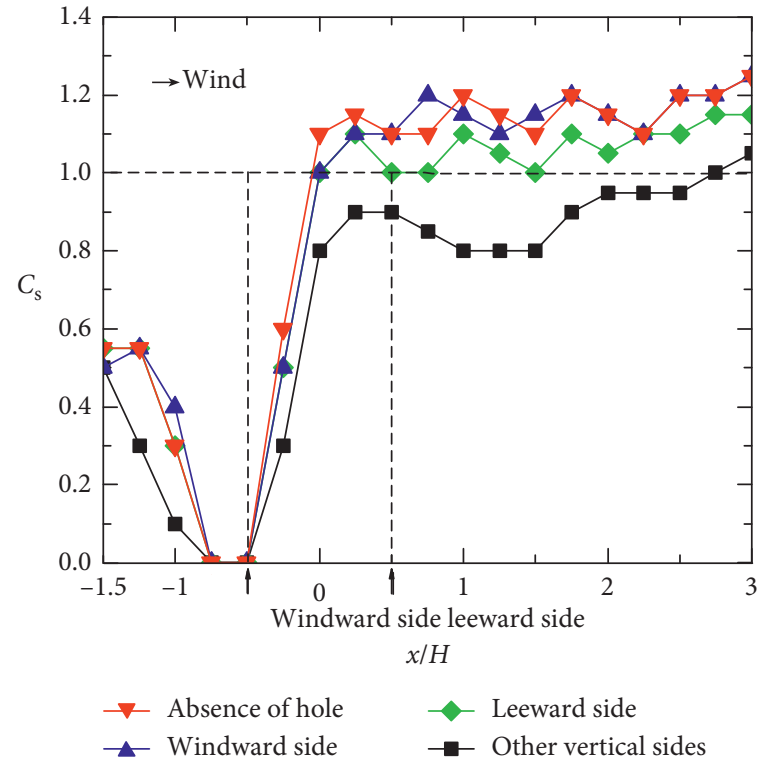

(b)

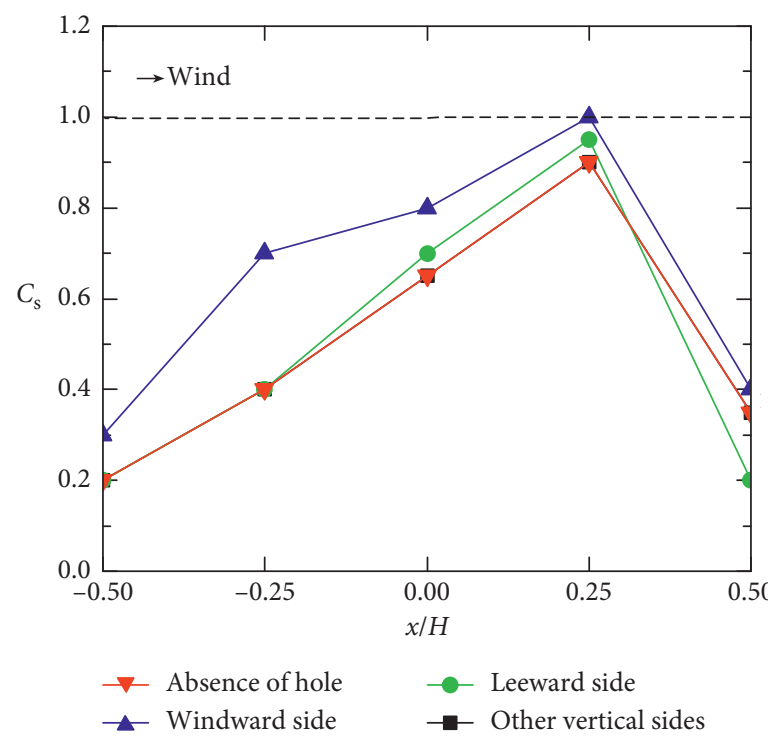

(c)

Figure 10: Snow distribution curve of a single cube: (a) along central and (b) longitudinal axes and (c) model surface.

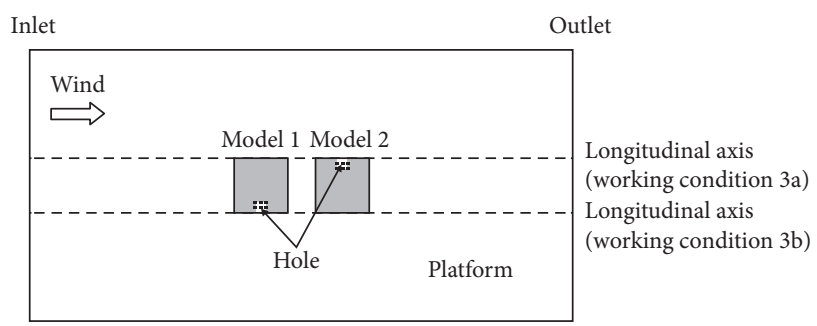

Figure 11: Three working conditions of snow depth coefficient of the longitudinal axis.

deposited in the middle of the two models, around the disturbed model and leeward area. As shown in Figure 12(a), when holes were respectant, wind change between the two models was more complicated. Unlike other conditions, snow depth coefficient of the central axis was first decreased and then increased, and its maximum value reached 1.4. Under this condition, the $C_{\mathrm{s}}$ value of the central axis in the leeward side $(x / H=-0.5)$ of the disturbance model was about $14.29 \%$ and $7.14 \%$ larger than those of the opposite position and the dislocation of the hole. Figure 12(b) shows that the value of $C_{\mathrm{s}}$ at the edge of the disturbance model rapidly increased from $0(x / H=-0.5)$ to $1.1 \sim 1.35(x /$ $H=-0.25)$. Due to interferences in the disturbance model, all of the snow was deposited at the edge $(x / H=1.5 \sim 2.5)$ of the disturbed model. The value of $C_{\mathrm{s}}$ varied in the range of 1.3 1.45. It suggested that doors, windows, and other emergency evacuation channels should be avoided in the 


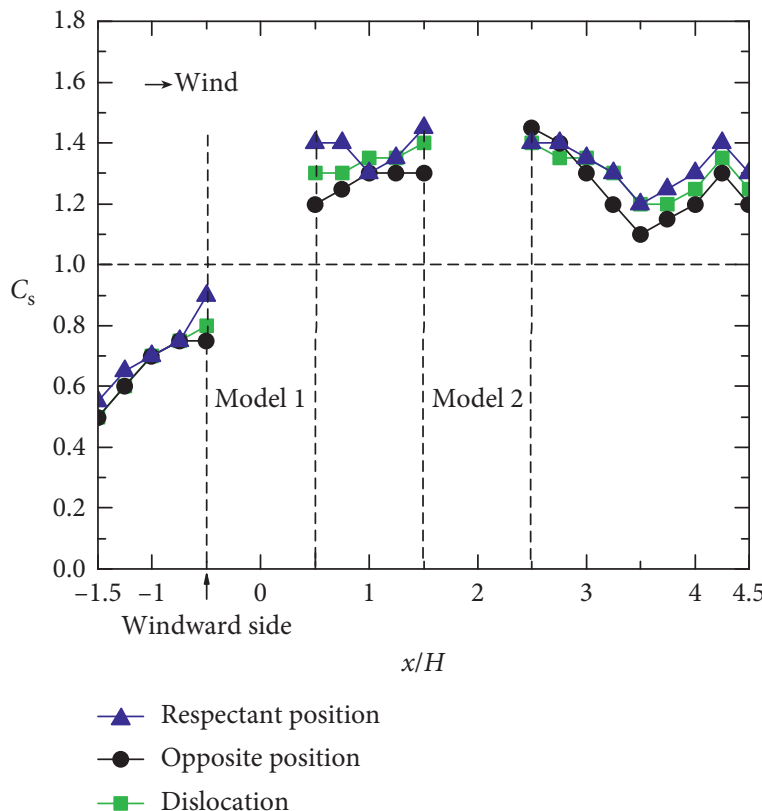

(a)

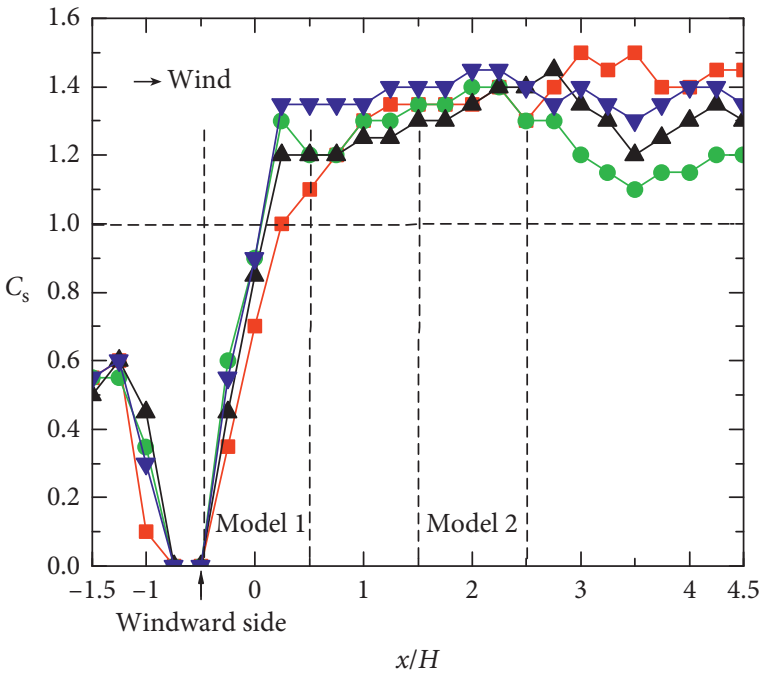

$\boldsymbol{\nabla}$ Working condition $1-$ Working condition 3a

$\leftarrow$ Working condition $2 \rightarrow$ Working condition $3 \mathrm{~b}$

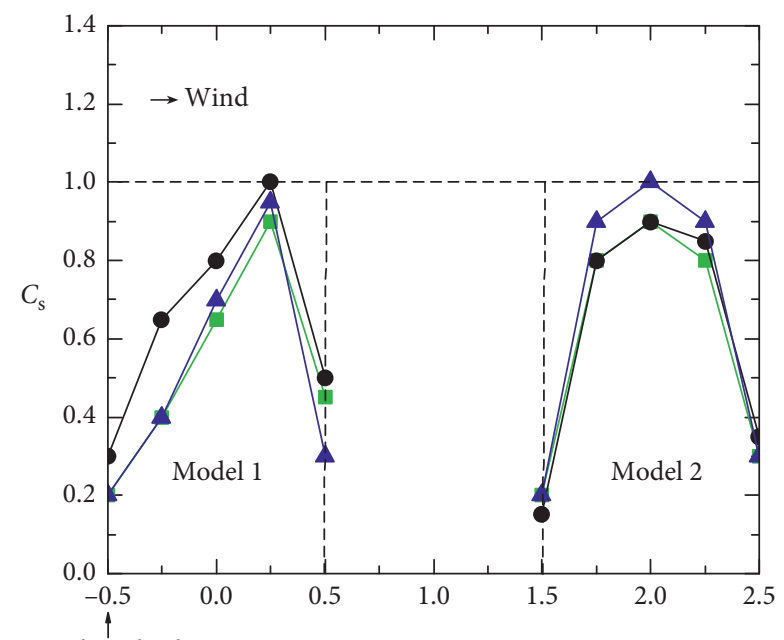

Windward side

(b)

$$
x / H
$$$$
\begin{aligned}
& - \text { Respectant position } \\
& - \text { - Opposite position } \\
& \rightarrow-\text { Dislocation }
\end{aligned}
$$

(c)

FIGURE 12: Snow distribution curve at different hole locations of the two models: snow distribution around the (a) central and (b) longitudinal axes as well as (c) surface area.

design. When holes were respectant, the value of $C_{\mathrm{s}}$ along the longitudinal axis at the leeward edge of the disturbance model was about $18.52 \%$, higher than that in working condition $3 \mathrm{~b}$, which indicated that snow distributions around the two models were relatively unfavorable for structures. When holes were dislocated, value differences along longitudinal axes on both sides of leeward area had to be considered seriously. Figure 12(c) shows that most of the snow on the roof was eroded. Surface snow erosion in the disturbance model was greater than that in the disturbed model due to blocking effect. In the disturbance model, the value of $C_{\mathrm{s}, \max }$ varied between 0.9 and 1 , and the maximum value of $C_{\mathrm{s}}$ appeared at $x / H=-0.25$. However, in the disturbed model, the value of $C_{\mathrm{s}}$ was large when $x / H$ varied between 1.75 and 2.25, the maximum value of $C_{\mathrm{s}}$ appeared at $x / H=2.0$, and the value of $C_{\mathrm{s}, \max }$ varied between 0.9 and 1 . At this time, the value of $C_{\mathrm{s}}$ reached its maximum, i.e., $C_{\mathrm{s}}=1$. In summary, respectant holes had an unfavorable effect on snow distribution in the surroundings and surfaces of the model. 


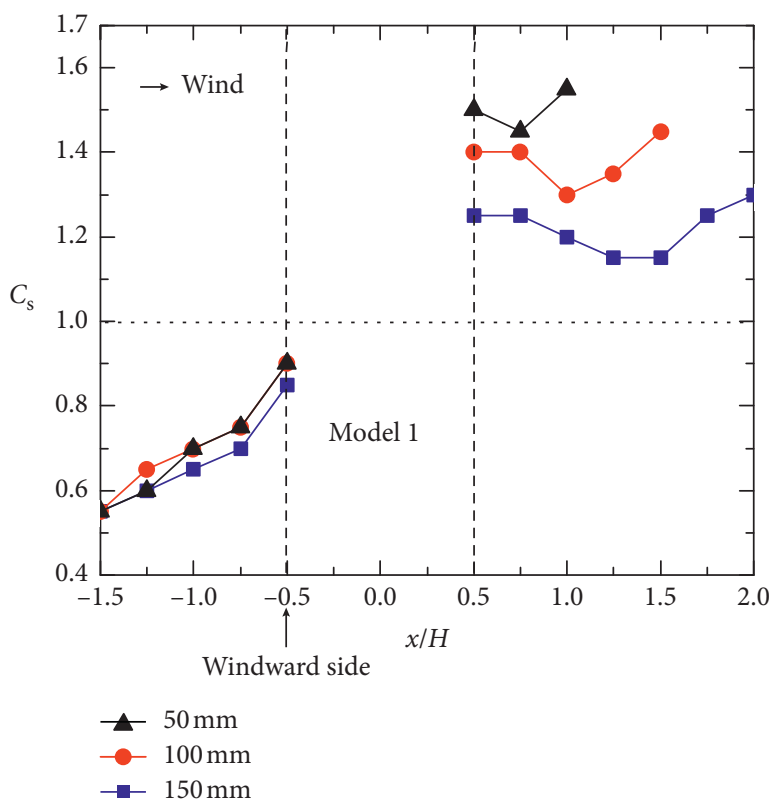

(a)

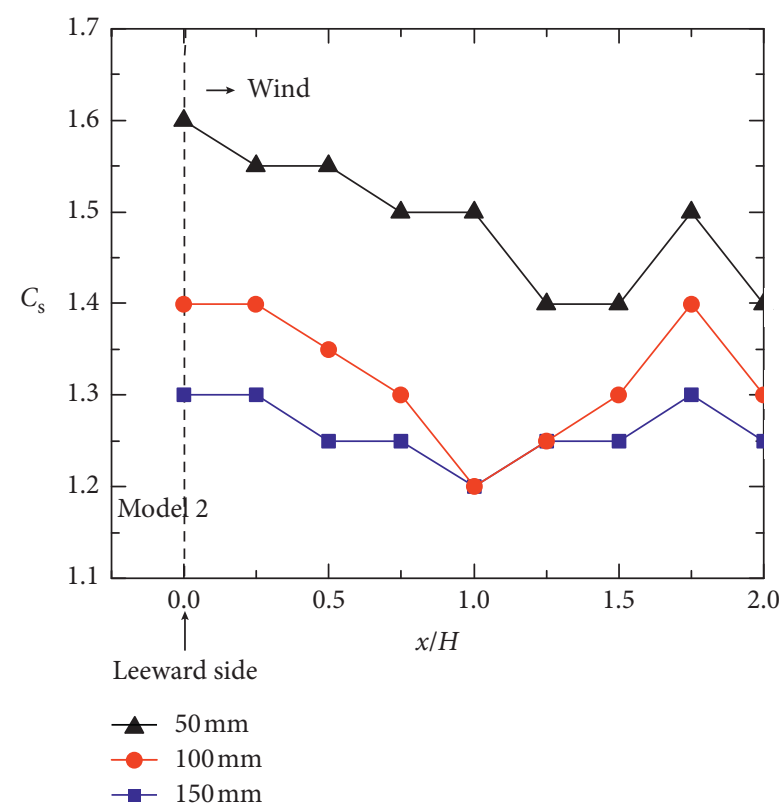

(b)

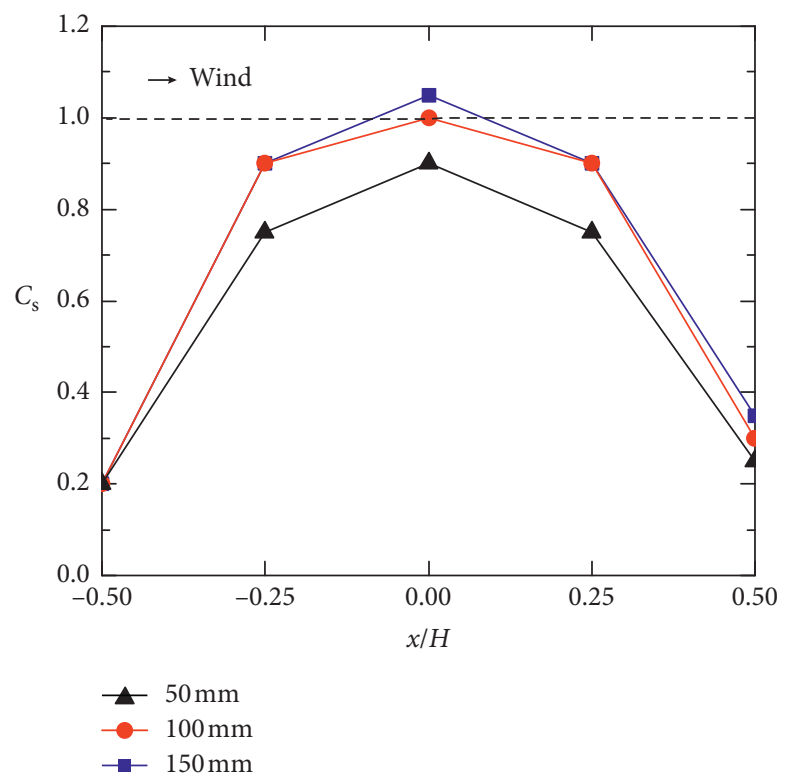

(c)

FIGURE 13: Snow distribution curves of (a) model 1 and (b) model 2 with different distances around their corresponding central axes and (c) surface area snow distribution of model 2.

4.3. Distance. When two cubes were respectant, the models were symmetric about the central axis. Therefore, snow distribution on the central axis was studied when the distance between two models was $50 \mathrm{~mm}, 100 \mathrm{~mm}$, and $150 \mathrm{~mm}$. The positions of the models are shown in Figure 8. Since the surface area snow distribution of model 1 was basically the same under the three mentioned conditions, as shown in Figure 12(c), only surface snow depth coefficient of model 2 was analyzed (Figure 13).

From Figure 13(a), it was seen that the change of $C_{s}$ under three conditions in the disturbance model (model 1) was basically the same on windward area $(x / H=-1.5 \sim-0.5)$.
The area of $C_{\mathrm{s}}$ between the two models first decreased and then increased. Due to wind flow around two holes, the values of $C_{\mathrm{s}}$ were quite different. The value of $C_{\mathrm{s}}$ at $50 \mathrm{~mm}$ spacing was about $16.67 \%$ higher than that at $150 \mathrm{~mm}$ at the leeward edge $(x / H=0.5)$ of the disturbance model. From Figure 13(b), the value of $C_{\mathrm{s}}$ at $50 \mathrm{~mm}$ spacing was $18.75 \%$ higher than that at $150 \mathrm{~mm}$ at the leeward edge $(x / H=0)$ of the disturbed model (model 2). The value of $C_{s}$ in leeward area first decreased, then increased and finally decreased, which was because snow was creeping. The difference between $50 \mathrm{~mm}$ and $150 \mathrm{~mm}$ spacings at $x / H=0.5$ was the largest (19.35\%). It was concluded from the aforementioned 
two figures that by decreasing the distance between the two models, the value of $C_{s}$ was increased. From Figure 13(c), it was observed that, due to the interference effect of the disturbance model, the snow distribution of surface area in the disturbed model was different from that in a single cube. The value of $C_{s, \max }$ appeared at the midpoint of the surface and was increased as the spacing was increased.

\section{Discussion}

Test equipment, snow materials, and human operation factors cause certain errors in wind tunnel test results of wind-induced snow distribution tests in holed structures. However, the results could still reveal certain rules. Therefore, based on the conclusions drawn from the experiments, different positions of the hole in holed structures and relative positions and spacing of different holes in adjacent holed structures were discussed.

Since the edges of cubes were blunt corners, wind was separated from side corners. Wind speed in these areas was significantly higher than that at the same height. This caused the wind to produce two large-scale horseshoe vortexes on the windward edges of cubes. Snow was completely eroded and carried around cube sides, and snow depth was sharply increased. Sudden change of snow is extremely influenced in structural safety. This situation occurred in the surroundings of single and disturbance structures, and the snow distribution patterns of disturbed structures were completely different from disturbance structures. Because incoming winds were no longer perpendicular to the windward side of the disturbed structure, the vortex of leeward area was not fully developed causing the airflow streamline to be deformed.

It was shown that holes had great influences on snow distribution patterns around holed structures. A pressure difference was created between the inside and outside of the hole causing wind pattern to change around it such that flow patterns varied with the orientation of hole resulting in different snow depth coefficients around the structures. A big difference was observed in snow distributions around the two-holed cubes at different relative positions. When the holes were respectant, wind speed at the hole was faster than that in other areas at the same height making wind patterns more complicated. Spacing was another important factor affecting snow distribution patterns between two holed structures. When distance was decreased, the mutual interference effect of the two structures became significant and snow depth coefficient around the structure increased, which was more unfavorable to the structures.

\section{Conclusions}

Wind tunnel tests were carried out on cubic samples to investigate snow distribution patterns considering the orientations of holed structures as well as relative positions and spacings of two-holed structures. The following conclusions were drawn:

(1) In the case of a small low-speed wind tunnel which satisfied Reynolds number and ignored Froude number, a difference between the measured and tested minimum values of snow depth coefficient along the central axis of the windward side of the cube was observed. The snow depth coefficients of the leeward side were different but their changing trends were similar. After analyzing possible reasons, it is believed that the small low-speed wind tunnel could be applied for wind tunnel tests of wind-induced snow distribution.

(2) The presence and location of the hole had significant effect on cube snow distribution. When the hole was on the other vertical sides, it had a favorable effect on snow distribution. Snow depth coefficient was different when the hole was located on the windward side. Under this condition, snow distribution was severely nonuniform with minimum and maximum values of 0 and 1.1, respectively.

(3) Snow pattern changes were more complicated when the holes of the two structures were respectant. Under this condition, snow depth coefficient firstly decreased and then increased between the areas of two-holed structures, and maximum snow depth coefficient reached 1.4. Snow depth around the structure decreased while maximum snow depth on the surface increased with the increase of distance between the structures.

(4) The standard cube snow distribution of holes was studied under different parameter values to provide a reasonable basis for the design of typical holed structures under the effect of wind and snow.

\section{Data Availability}

The data used to support the findings of this study are available from the corresponding author upon request.

\section{Conflicts of Interest}

The authors declare that they have no conflicts of interest.

\section{Acknowledgments}

This research was supported by the National Natural Science Foundation of China (grant no. U1261122).

\section{References}

[1] Y. Anno, "Requirements for modeling of a snowdrift," Cold Regions Science and Technology, vol. 8, no. 3, pp. 241-252, 1984.

[2] J. D. Iversen, "Drifting-snow similitude-transport-rate and roughness modeling," Journal of Glaciology, vol. 26, no. 94, pp. 393-403, 1980.

[3] J. D. Iversen, "Comparison of wind-tunnel model and fullscale snow fence drifts," Journal of Wind Engineering and Industrial Aerodynamics, vol. 8, no. 3, pp. 231-249, 1981.

[4] R. J. Kind, "Snowdrifting: a review of modelling methods," Cold Regions Science and Technology, vol. 12, no. 3, pp. 217228, 1986. 
[5] R. J. Kind, "A critical examination of the requirements for model simulation of wind-induced erosion/deposition phenomena such as snow drifting," Atmospheric Environment (1967), vol. 10, no. 3, pp. 219-227, 1976.

[6] D. H. Kim, K. C. S. Kwok, D. J. Smedley et al., "Modelling of snowdrift around prismatic buildings for Antarctic environment," International Journal of Offshore and Polar Engineering, vol. 2, no. 1, pp. 73-79, 1992.

[7] J. D. Iversen, "Comparison of snowdrift modeling criteria: commentary on "Application of Anno's modeling conditions to outdoor modeling of snowdrifts", "Cold Regions Science and Technology, vol. 9, no. 3, pp. 259-265, 1984.

[8] J. A. Peterka and W. S. Esterday, "Roof design snow loads by wind tunnel test and analysis," in Proceedings of the Structures 2004: At Building on the Past, Securing the Future, pp. 1-9, ASCE, Nashville, TN, USA, May 2004.

[9] M. O’Rourke, A. DeGaetano, and J. D. Tokarczyk, "Snow drifting transport rates from water flume simulation," Journal of Wind Engineering and Industrial Aerodynamics, vol. 92, no. 14-15, pp. 1245-1264, 2004.

[10] Z. X. Liu, Z. X. Yu, F. Zhu et al., "An investigation of snow drifting on flat roofs: wind tunnel tests and numerical simulations," Cold Regions Science and Technology, vol. 162, pp. 74-87, 2019.

[11] Z. X. Yu, F. Zhu, R. Z. Cao et al., "Wind tunnel tests and CFD simulations for snow redistribution on 3D stepped flat roofs," Wind and Structures, vol. 28, no. 1, pp. 31-47, 2019.

[12] X. Zhou, J. Hu, and M. Gu, "Wind tunnel test of snow loads on a stepped flat roof using different granular materials," Natural Hazards, vol. 74, no. 3, pp. 1629-1648, 2014.

[13] X. Li, Research on Snowdrifting on Building Roof and around Building, Tongji University, Shanghai, China, 2011.

[14] W. H. Wang, H. L. Liao, and M. S. Li, "Wind tunnel test on wind-induced roof snow distribution," Journal of Building Structures, vol. 35, no. 5, pp. 135-141, 2014, in Chinese.

[15] X. Zhou, L. Kang, X. Yuan, and M. Gu., "Wind tunnel test of snow redistribution on flat roofs," Cold Regions Science and Technology, vol. 127, pp. 49-56, 2016.

[16] D. J. Smedley, K. C. S. Kwok, and D. H. Kim, "Snowdrifting simulation around Davis Station workshop, Antarctica," Journal of Wind Engineering and Industrial Aerodynamics, vol. 50, pp. 153-162, 1993.

[17] S. Oikawa, T. Tomabechi, and T. Ishihara, "One-day observations of snowdrifts around a model cube," Journal of Snow Engineering of Japan, vol. 15, no. 4, pp. 283-291, 1999.

[18] Q. K. Ishihara, S. B. Zhao, S. J. Meng et al., "Codes comparison and wind tunnel simulation of wind-induced snowdrift," Engineering Mechanics, vol. 32, no. 1, pp. 50-56, 2015, in Chinese.

[19] J. H. M. Beyers and T. M. Harms, "Outdoors modelling of snowdrift at SANAE IV research station, Antarctica," Journal of Wind Engineering and Industrial Aerodynamics, vol. 91, no. 4, pp. 551-569, 2003.

[20] R. J. Kind and S. B. Murray, "Saltation flow measurements relating to modeling of snowdrifting," Journal of Wind Engineering and Industrial Aerodynamics, vol. 10, no. 1, pp. 89-102, 1982. 


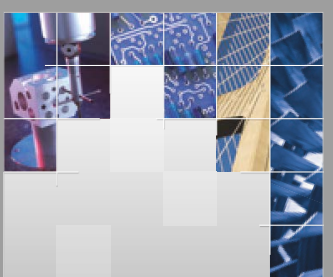

\section{Enfincering}
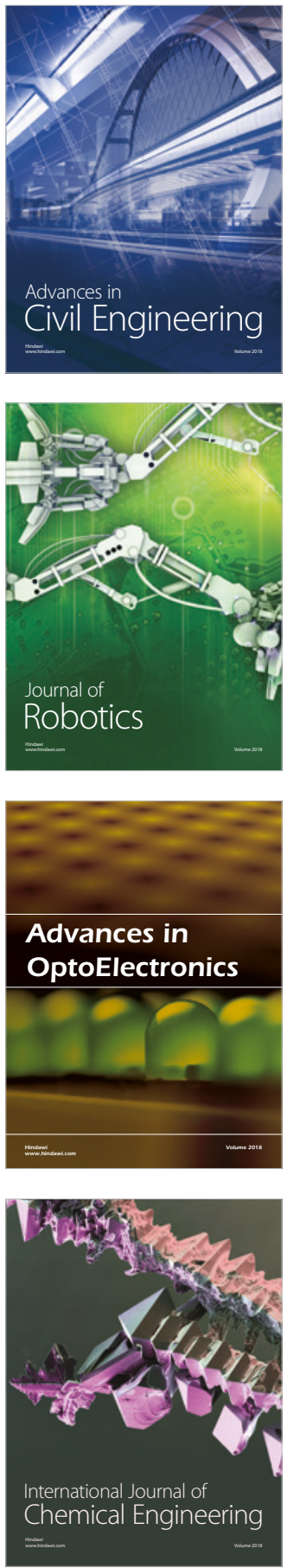

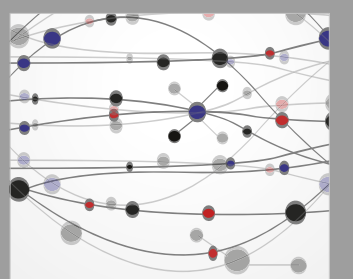

\section{Rotating \\ Machinery}

The Scientific World Journal

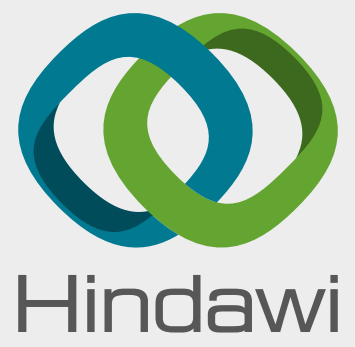

Submit your manuscripts at

www.hindawi.com
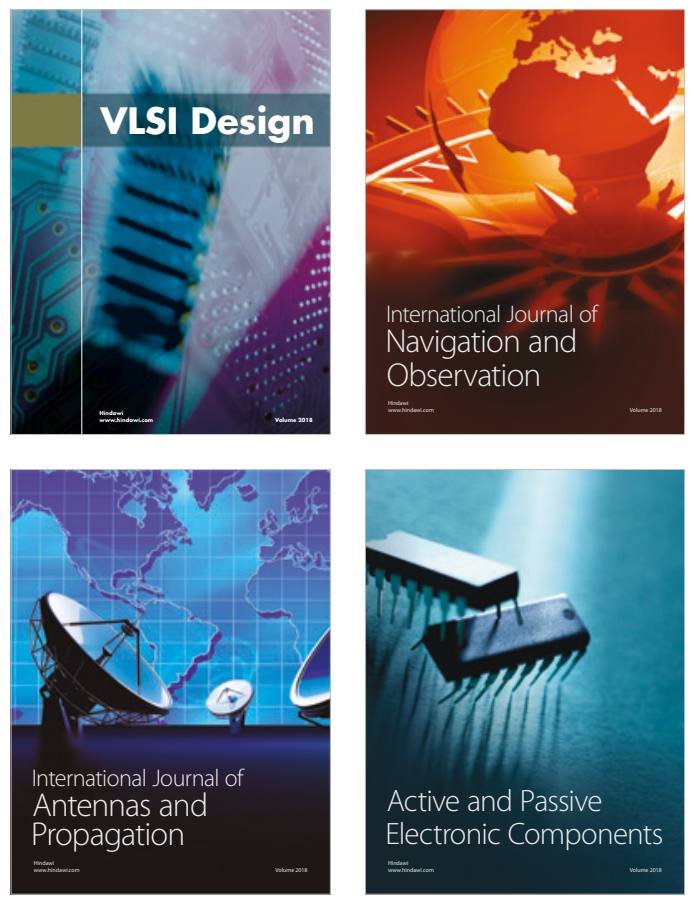
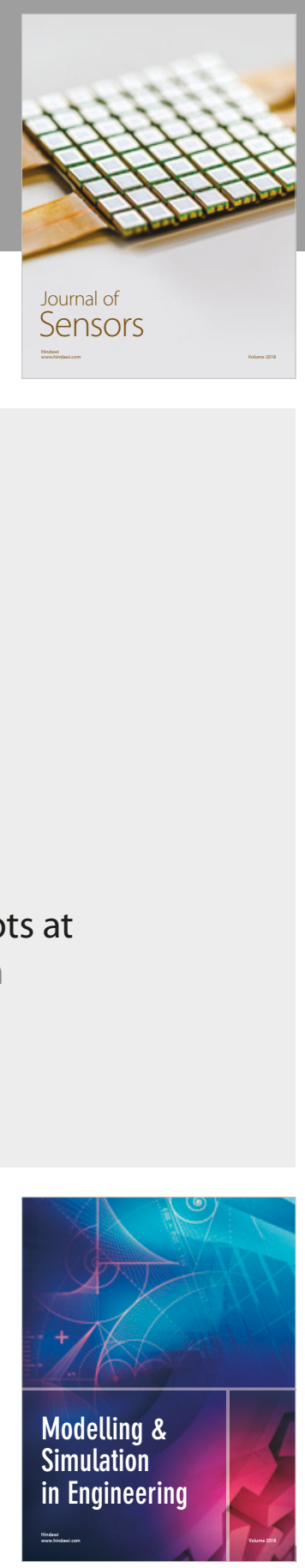

\section{Advances \\ Multimedia}
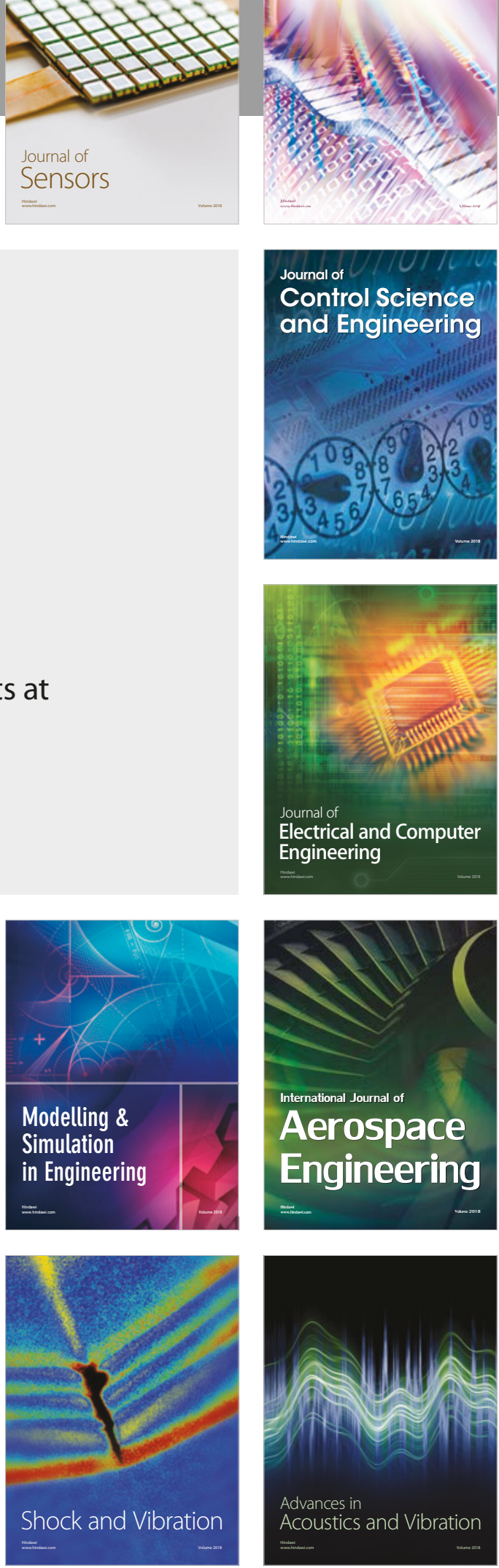